\title{
Utility of Nerve Conduction Studies for Carpal Tunnel Syndrome by Family Medicine, Primary Care, and Internal Medicine Physicians
}

\author{
J. Thomas Megerian, Xuan Kong, and Shai N. Gozani
}

Introduction: Nerve conduction studies (NCS) are increasingly being performed at the point-of-service by family medicine, primary care, and internal medicine (FM/PCP/IM) physicians. Carpal tunnel syndrome (CTS) is a common neuropathy often diagnosed with the aid of NCS.

Methods: A retrospective analysis of a point-of-service NCS data registry was conducted; 1190 patients who underwent NCS by 613 FM/PCP/IM physician practices, for evaluation of CTS were analyzed. Utility measures included demographic and electrophysiological characteristics of study population, adherence to evidence-based testing guidelines, and relevance of diagnostic outcomes.

Results: Tested patients tended to be over 40 , female, and overweight or obese. The median nerve distal motor latency was $4.4 \pm 1.2 \mathrm{~ms} ; \mathbf{9 2 . 6 \%}$ of studies met the testing guideline; $30.5 \%$ of tested limbs yielded normal results; $53.1 \%$ CTS; $5.4 \%$ ulnar neuropathy; and $11.0 \%$ nonspecific upper extremity neuropathy.

Discussion: This study demonstrated that point-of-service NCS by FM/PCP/IM physicians for CTS was applied to appropriate patient subpopulations, was performed in accordance with evidence-based testing parameters, and generated relevant diagnostic outcomes. (J Am Board Fam Med 2007;20:60-4.)

Electrodiagnostic procedures include nerve conduction studies (NCS) and/or needle electromyography (nEMG). They have historically been performed by physicians with neurology or physical and rehabilitative medicine (PM\&R) training. However, an analysis of a health insurance claims database showed that, in 1999, as many as one quarter of electrodiagnostic procedures were performed by physicians with other backgrounds. ${ }^{1}$ nEMG causes significant patient discomfort, ${ }^{2}$ has a risk of adverse events, ${ }^{2}$ and is subjective. ${ }^{3}$ It is therefore often performed by physicians with specific training. NCS are objective, non-invasive, and generally standardized ${ }^{4}$ and may therefore be performed and interpreted by a wide array of physicians. Expanded access to NCS has the potential to

This article was externally peer reviewed.

Submitted 30 June 2006; revised 22 August 2006; accepted 24 August 2006.

From NeuroMetrix, Inc.

Funding: This study was supported by NeuroMetrix, Inc.

Conflict of interest: Drs. J. Thomas Megerian, Xuan Kong, and Shai Gozani are employees of NeuroMetrix, Inc.

Corresponding author: Xuan Kong, NeuroMetrix, Inc., 62 Fourth Avenue, Waltham, MA 02451 (E-mail: Xuan_ Kong@neurometrix.com) improve outcomes. ${ }^{5}$ However, constraints on the availably of NCS have probably limited its usage, ${ }^{5,6}$ particularly early in the episode of care.

Increasingly sophisticated automation technology is being incorporated into devices that perform NCS. ${ }^{7}$ With these advances, a wider array of physicians, including those in family medicine, primary care, and internal medicine (FM/PCP/IM), are integrating NCS into their clinical practice. Like other diagnostic procedures previously performed by specialists, ${ }^{8}$ the utility of NCS performed by non-specialists has been debated. ${ }^{1}$ However, the arguments have been opinion based, ${ }^{9}$ which do not constitute evidence-based medicine. ${ }^{4}$

Population-based evaluations of diagnostic procedures are often hampered by the absence of a reliable reference standard with which to assess accuracy and difficulty obtaining clinical outcome data. Carpal tunnel syndrome (CTS) is the most common focal neuropathy and a major cause of disability in the United States. ${ }^{10}$ The utility of NCS, which includes objective confirmation of nerve involvement, grading of severity, and exclusion of polyneuropathy, is well accepted ${ }^{11}$ and evidencebased diagnostic ${ }^{12}$ and treatment guidelines ${ }^{13}$ exist. 
Table 1. Definition of Electrophysiological Diagnostic Outcomes

\begin{tabular}{|c|c|c|c|c|c|}
\hline \multirow[b]{2}{*}{ Outcome* } & \multicolumn{2}{|c|}{ Median Nerve $†$} & \multicolumn{2}{|c|}{ Ulnar Nerve† } & \multirow{2}{*}{$\begin{array}{c}\text { Combined } \\
\text { MUD }\end{array}$} \\
\hline & DML & DSL & DML & DSL & \\
\hline Normal & Normal & Normal & Normal & Normal & Normal \\
\hline CTS $\ddagger$ & Abnormal & Abnormal & Normal & Normal & Abnormal \\
\hline Ulnar\$ & Normal & Normal & Abnormal & Abnormal & Normal \\
\hline Nonspecificd & Abnormal & Abnormal & Abnormal & Abnormal & Normal \\
\hline
\end{tabular}

* Normal, no neuropathy; CTS, carpal tunnel syndrome; ulnar, ulnar neuropathy; nonspecific, nonspecific upper extremity neuropathy affecting both median and ulnar nerves.

† DML, distal motor latency; DSL, distal sensory latency; MUD, median-ulnar distal sensory latency difference; normal DML or DSL, $<97.5 \%$ of reference population; normal MUD, <90\% of reference population; MUD threshold percentile lower than DML and DSL to match clinical practice.

‡ One of median nerve DML, median nerve DSL, or MUD must be abnormal.

$\$$ One of ulnar nerve DML or DSL must be abnormal.

II One of median nerve DML or DSL must be abnormal and one of ulnar nerve DML or DSL must be abnormal.

As a result, CTS is an appropriate disease model for evaluating the quality and efficacy of diagnostic testing. In this study, a population-based analysis of FM/PCP/IM physician utilization of NCS for the assessment of CTS was conducted. Utilization measures included demographic characteristics of the tested population, nerve conduction values, adherence to evidence-based testing guidelines, and relevance of diagnostic outcomes.

\section{Methods}

All NCS performed over 10 consecutive days (January 2006) using an automated NCS instrument (NC-stat; NeuroMetrix, Inc., Waltham, MA) linked to a data registry ${ }^{7}$ were analyzed retrospectively. Physicians using the instrument during this period were unaware of the eventual research use of the data and were therefore blinded to the study. Instrument operation and accuracy in detecting CTS have been described. ${ }^{7,15,17}$ In brief, this instrument performs conventional motor and sensory NCS. It automates the technical steps of a NCS including electrode placement, skin surface temperature correction, determination of nerve stimulation intensity, and analysis of the evoked neuroelectrical responses. The system comprises "biosensors," an electronic monitor, and a report generation system. The registry stores all electrophysiological data including raw waveforms and limited demographic information (age, height, weight, and gender). The NCS tests are typically performed by office clinical staff (ie, allied health professionals and nurses) that undergo 1 day of on-site training by the manufacturer. The instru- ment and the data registry have automated quality assurance software that confirms and tracks ongoing staff competence. No technical or clinical retraining was provided in advance of the data analysis period. Each study is coded with the primary clinical indication for the evaluation, from among a list that includes CTS, cubital tunnel syndrome, C8 or T1 radiculopathy, back pain, leg pain, sciatica, diabetic polyneuropathy, unspecified polyneuropathy, occupational screening, and unspecified indication. Inclusion criteria were studies 1) coded for CTS, 2) performed by a provider coded as family medicine, primary care, internal medicine, rheumatology, or endocrinology, and 3) including data for at least one median or ulnar nerve. There were no exclusion criteria. This study was performed under Institutional Review Board protocol no. 99000266 (Copernicus Group, Cary, NC).

A patient study was defined as strictly compliant with the evidence-based CTS testing guideline ${ }^{12}$ if it included at least one limb with both median and ulnar distal sensory measurements. A second less restrictive definition was 2 or more upper extremity distal sensory measurements. The later definition was required for comparison to a prior study ${ }^{14}$ based on an insurance claims database. Further data analysis was performed with individual limbs as the unit of evaluation. This analysis cohort was defined as the subset of limbs for which both median and ulnar nerve data were available. Four diagnostic outcomes, summarized in Table 1, were defined based on this electrophysiological data. Abnormalities were defined relative to the upper (latencies) or lower (amplitudes) limits of normal after adjust- 


\begin{tabular}{lcccc}
\hline Age Range $(\%$ Total $)$ & Gender $(\%$ Female $)$ & Height $(\mathrm{cm})^{*}$ & Weight $(\mathrm{kg})^{*}$ & BMI $\left(\mathrm{kg} / \mathrm{m}^{2}\right)^{*}$ \\
\hline$<30(5.9)$ & 73.2 & $168(10)$ & $84(25)$ & $29.5(7.0)$ \\
$30-39(13.4)$ & 71.1 & $167(10)$ & $86(23)$ & $30.5(7.2)$ \\
$40-49(21.3)$ & 71.7 & $167(10)$ & $87(23)$ & $31.0(7.5)$ \\
$50-59(22.8)$ & 71.6 & $166(10)$ & $86(22)$ & $31.0(7.4)$ \\
$60-69(15.7)$ & 71.1 & $165(11)$ & $85(19)$ & $31.2(6.2)$ \\
$\geq 70(20.8)$ & 71.8 & $163(10)$ & $76(17)$ & $28.4(5.9)$ \\
\hline
\end{tabular}

* Mean (standard deviation).

ing electrophysiological measurements for skin surface temperature, patient height, and age. ${ }^{15}$ Each limb was assigned a diagnostic outcome (normal, CTS, ulnar neuropathy, or nonspecific upper extremity neuropathy) using these definitions.

\section{Results}

The initial data set consisted of NCS from 1190 patients performed by 613 different FM/PCP/IM physician practices. Mean patient age was $53.3 \pm$ 13.3 years with $80.7 \% 40$ years or older, and $27.8 \%$ 65 years or older (see Table 2 for study group demographics). Of the patients, $71.6 \%$ were female, and the mean body mass index (BMI) was $30.3 \pm 6.9 \mathrm{~kg} / \mathrm{m}^{2}$ with $9.1 \%$ morbidly obese (BMI 240). Studies in $971(81.6 \%)$ and 1102 (92.6\%) patients adhered to the strict and less restrictive CTS testing guidelines, respectively, and were further analyzed. Among these studies, 1585 limbs had both median and ulnar nerve data and represented the analysis cohort. The median nerve distal motor latency (DML) was $4.4 \pm 1.2 \mathrm{~ms}$ (8 of 1585 limbs did not have a median nerve DML). Among limbs in the analysis cohort, 483 (30.5\%) had normal NCS, 842 (53.1\%) indicated CTS, 86 (5.4\%) identified an ulnar neuropathy, and 174 (11.0\%) limbs were labeled a nonspecific neuropathy involving both the median and ulnar nerves.

\section{Discussion}

In a commercial insurance claims analysis of NCS procedures, Dillingham and colleagues showed that up to $25 \%$ of physician-supervised studies were performed by physicians who were not neurologists or $\mathrm{PM} \& \mathrm{R}$ physicians. ${ }^{1}$ There are clinical, ${ }^{5}$ patient satisfaction, ${ }^{15,20}$ and potentially economic reasons for expanding access to reliable NCS, particularly early in the episode of care. Given these factors, it is likely that point-of-service NCS by FM/PCP/IM physicians will continue expanding.

This study showed that NCS was used by FM/ PCP/IM physicians for evaluation of CTS in patients that were typically female, $>40$ years of age, and overweight or obese. These demographic features are strongly associated with a higher risk of CTS. ${ }^{18}$ The average median nerve DML was 4.4 ms, which was 2.3 normal deviates above the mean value for asymptomatic disease free limbs. These demographic and electrophysiological characteristics suggest that for CTS, point-of-service NCS was applied to the appropriate patient subgroup.

Practitioner adherence to accepted clinical guidelines has been used as an outcome measure when evaluating technological advances ${ }^{16}$ and procedure performance by primary care specialists. ${ }^{8}$ In this study, $81.6 \%$ of NCS studies performed by FM/PCP/IM physicians, for evaluation of CTS, met the strict evidence-based testing guideline. ${ }^{12}$ Using a less restrictive definition adopted by Storm and colleagues, ${ }^{14} 91.6 \%$ satisfied the guideline. Both metrics are comparable to $86.4 \%$ adherence by neurologists and $\mathrm{PM} \& \mathrm{R}$ physicians reported in the Storm study, which was based on Medicare patients who went on to carpal tunnel release surgery. ${ }^{14}$ The high rate of compliance by FM/ PCP/IM physicians employing point-of-service NCS confirms that their patients received standard-of-care testing.

An important measure of diagnostic value is the diversity among the diagnostic outcomes. It is intuitively clear that a test yielding the same reading in most individuals within a population is not informative because it is unlikely to result in differentiated treatment. Conversely, if a variety of diagnostic outcomes occur, then the test results partition the population into groups that may benefit from different interventions. In this study, CTS 
was identified in $53.1 \%$ of tested limbs; in another $30.5 \%$ the study was normal. Therefore in $83.6 \%$ of tested limbs, the specific diagnostic question of whether the patient had or did not have CTS was addressed by the point-of-service NCS. This high rate suggests that pretest patient selection led to clinically relevant studies. A potential criticism of point-of-service NCS by FM/PCP/IM physicians was that the studies would be mostly used in normal patients for which testing was not clinically informative or many studies would yield nonspecific results. These theoretical arguments were contradicted by the results of this study. In fact, the results were similar to a claims-based analysis of CTS testing by neurologists and PM\&R physicians. ${ }^{14}$ Approximately $11 \%$ of the limbs were labeled with a nonspecific neuropathy. This classification was based entirely on abnormalities of ipsilateral median and ulnar nerve data for each limb. Data from the contralateral limb and lower extremities were not considered and would be expected to reduce the number of nonspecific neuropathies.

The aforementioned conclusions are predicated on an assumption of instrument accuracy in detecting CTS. The validity of automated distal latency measurements, in the median and ulnar nerves, has been confirmed by high correlation (coefficients typically 0.85 to 0.95 ) to blinded traditional electromyography laboratories. ${ }^{15,17,19-21}$ These coefficients are comparable to inter-examiner correlation between board certified electromyographers. ${ }^{22}$ In 3 studies meeting class I or II evidence-based critieria, ${ }^{15,17,21}$ the positive and negative likelihood ratios for CTS ranged from 8.6 to $>10$ and 4.3 to 8.6, respectively (likelihood ratios calculated from reported sensitivity, specificity, and raw agreement). These likelihood ratios represent clinically meaningful changes from pretest to post-test disease probability in terms of both ruling-in and rulingout CTS. ${ }^{23}$

This study had several limitations. First, data from a single NCS registry, associated with a specific electrodiagnostic instrument, were analyzed. This might lead to selection bias because participating physicians had greater electrodiagnostic knowledge than the general FM/PCP/IM physician population. Second, only studies coded for CTS testing were analyzed. It was possible that patients coded for CTS had the most distinct clinical presentations and therefore electrodiagnostic results might be expected to be most definitive. Examination of studies coded for ulnar neuropathies and other upper extremity neuropathies would be valuable. However, approximately $71 \%$ of all upper extremity studies during the evaluation period were coded for CTS. Third, studies identified as normal or CTS were defined as diagnostically specific although subsequent clinical outcomes were not measured. CTS is a well defined neurophysiological entity with a treatment pathway guided by confirmation of a focal median neuropathy at the wrist and determination of its severity. ${ }^{13}$ For example, abnormal median nerve DML measured by this electrodiagnostic instrument was predictive of post carpal tunnel release improvement in median nerve function. ${ }^{17}$ As a result, definitive detection of CTS or its exclusion (ie, normal diagnostic outcome) was a valid and useful clinical outcome. Fourth, this study did not evaluate FM/ PCP/IM physician clinical interpretation of the NCS data. There is no "gold standard" for neurophysiological diagnosis as subjectivity and bias lead to limited inter-examiner interpretation agreement even among physicians regarded as experts. ${ }^{3,24} \mathrm{Me}-$ dium and long-term clinical outcomes such as symptom resolution and patient satisfaction could be used to evaluate the quality of diagnostic interpretations; however, such studies have yet to be performed for traditional electrodiagnostic studies by neurologists and $\mathrm{PM} \& \mathrm{R}$ physicians.

\section{References}

1. Dillingham TR, Pezzin LE, Rice JB. Electrodiagnostic services in the United States. Muscle Nerve 2004;29:198-204.

2. Al-Shekhlee A, Shapiro BE, Preston DC. Iatrogenic complications and risks of nerve conduction studies and needle electromyography. Muscle Nerve 2003; 27:517-26.

3. Johnsen B, Fuglsang-Frederiksen A, Vingtoft S, et al. Inter- and intraobserver variation in the interpretation of electromyographic tests. Electroencephalogr Clin Neurophysiol 1995;97:432-43.

4. England JD, Gronseth GS, Franklin G, et al. Distal symmetrical polyneuropathy: definition for clinical research. Muscle Nerve 2005;31:113-23.

5. Perkins BA, Bril V. Diabetic neuropathy: a review emphasizing diagnostic methods. Clin Neurophysiol 2003;114:1167-75.

6. Duncan KH, Lewis RC Jr., Foreman KA, Nordyke MD. Treatment of carpal tunnel syndrome by members of the American Society for Surgery of the 
Hand: results of a questionnaire. J Hand Surg [Am] 1987;12:384-91.

7. Gozani SN, Fisher MA, Kong X, Megerian JT, Rutkove SB. Electrodiagnostic automation: principles and practice. Phys Med Rehabil Clin N Am 2005; 16:1015-32.

8. Edwards JK, Norris TE. Colonoscopy in rural communities: can family physicians perform the procedure with safe and efficacious results? J Am Board Fam Pract 2004;17:353-8.

9. American Association of Neuromuscular and Electrodiagnostic Medicine. Proper performance and interpretation of electrodiagnostic studies. Muscle Nerve 2006;33:436-9.

10. Blanc PD, Faucett J, Kennedy JJ, Cisternas M, Yelin E. Self-reported carpal tunnel syndrome: predictors of work disability from the National Health Interview Survey Occupational Health Supplement. Am J Ind Med 1996;30:362-8.

11. Rempel D, Evanoff B, Amadio PC, et al. Consensus criteria for the classification of carpal tunnel syndrome in epidemiologic studies. Am J Public Health 1998;88:1447-51.

12. Jablecki CK, Andary MT, Floeter MK, et al. Practice parameter: electrodiagnostic studies in carpal tunnel syndrome. Report of the American Association of Electrodiagnostic Medicine, American Academy of Neurology, and the American Academy of Physical Medicine and Rehabilitation. Neurology 2002;58: 1589-92.

13. Katz JN, Simmons BP. Clinical practice. Carpal tunnel syndrome. N Engl J Med 2002;346:1807-12.

14. Storm S, Beaver SK, Giardino N, et al. Compliance with electrodiagnostic guidelines for patients undergoing carpal tunnel release. Arch Phys Med Rehabil 2005;86:8-11.

15. Leffler CT, Gozani SN, Cros D. Median neuropathy at the wrist: diagnostic utility of clinical findings and an automated electrodiagnostic device. J Occup Environ Med 200;42:398-409.
16. Garg AX, Adhikari NK, McDonald H, et al. Effects of computerized clinical decision support systems on practitioner performance and patient outcomes: a systematic review. JAMA 2005;293:1223-38.

17. Rotman MB, Enkvetchakul BV, Megerian JT, Gozani SN. Time course and predictors of median nerve conduction after carpal tunnel release. J Hand Surg [Am] 2004;29(3):367-72.

18. Becker J, Nora DB, Gomes I, et al. An evaluation of gender, obesity, age and diabetes mellitus as risk factors for carpal tunnel syndrome. Clin Neurophysiol 2002;113:1429-34.

19. Vinik AI, Emley MS, Megerian JT, Gozani SN. Median and ulnar nerve conduction measurements in patients with symptoms of diabetic peripheral neuropathy using the NC-stat system. Diabetes Technol Ther 2004;6:816-24.

20. Elkowitz SJ, Dubin NH, Richards BE, Wilgis EF. Clinical utility of portable versus traditional electrodiagnostic testing for diagnosing, evaluating, and treating carpal tunnel syndrome. Am J Orthop 2005; 34:362-4.

21. Kong X, Gozani SN, Hayes MT, Weinberg DH. NC-stat sensory nerve conduction studies in the median and ulnar nerves of symptomatic patients. Clin Neurophysiol 2006;117:405-13.

22. Salerno DF, Werner RA, Albers JW, Becker MP, Armstrong TJ, Franzblau A. Reliability of nerve conduction studies among active workers. Muscle Nerve 1999;22:1372-9.

23. Jaeschke R, Guyatt GH, Sackett DL. Users' guides to the medical literature. III. How to use an article about a diagnostic test. B. What are the results and will they help me in caring for my patients? The Evidence-Based Medicine Working Group. JAMA 1994;271:703-7.

24. Kendall R, Werner RA. Interrater reliability of the needle examination in lumbosacral radiculopathy. Muscle Nerve. 2006;34:238-41. 